\title{
Combined intra-tendinous injection of Platelet Rich Plasma and bevacizumab accelerates and improves healing compared to Platelet Rich Plasma in tendinosis: comprehensive assessment on a rat model
}

\author{
Benjamin Dallaudiere ${ }^{1}$ \\ Olivier Zurlinden² \\ Anne Perozziello 3 \\ Lydia Deschamps ${ }^{4}$ \\ Ahmed Larbi ${ }^{5}$ \\ Liliane Louedec ${ }^{6}$ \\ Lionel Pesquer ${ }^{1}$ \\ Yohan Benayoun ${ }^{1}$ \\ Alain Silvestre ${ }^{1}$ \\ Jean Michel Serfaty 6
}

1 Centre d'Imagerie Ostéo-articulaire, Clinique du Sport de Bordeaux-Mérignac Département d'Imagerie Musculo-squeletrique, Centre Hospitalier Universitaire Pellegrin Bordeaux, France

2 Département d'Imagerie Musculo-squeletrique, Centre Hospitalier Universitaire Bichat, Paris, France

3 Unité de recherche Clinique, Paris Nord, Centre Hospitalier Universitaire Bichat, Paris, France

4 Département d'Anatomopathologie, Centre Hospitalier Universitaire Bichat, Paris, France

5 Département d'Imagerie Musculo-squeletrique, Cliniques Universitaires Saint Luc, Bruxelles, Belgium

6 Unité INSERM U698,Centre Hospitalier Universitaire Bichat, Paris, France

Corresponding author:

Benjamin Dallaudiere

Centre d'Imagerie Ostéo-articulaire, Clinique du Sport de Bordeaux-Mérignac Département d'Imagerie Musculo-squeletrique, Centre Hospitalier Universitaire Pellegrin

Place Amélie Léon Rabat

33000 Bordeaux, France

E-mail: benjamin.dallaudiere@gmail.com

\section{Summary}

Purpose: the aim of our study was to assess the potential of combined intratendinous injection of an anti-angiogenic drug: bevacizumab (AA) and Platelet Rich Plasma (PRP) to treat tendinopathy in a murine model of patellar and Achilles tendinopathy, and to evaluate its local toxicity. Material and method: twenty rats (80 patellar and Achilles tendons) were used for the study. We induced tendinosis $(T+)$ in 80 tendons (patellar $=40$ and Achilles $=40$ ) by injecting under ultrasonogra- phy (US) guidance Collagenase $1{ }^{\circledR}$ (day $0=\mathrm{D} 0$ ). Clinical examination was performed at D3, immediately followed by either PRP and AA (AAPRPT+, $\mathrm{n}=40$ ) or PRP (PRPT+ $\mathrm{n}=40$, control) US-guided intratendinous injection. Follow-up at D6, D18 and D25 using clinical, US and histology, and comparison between the 2 groups were performed. To study AA+PRP toxicity, we looked for necrosis or rupture on the 40 AAPRPT+.

Results: all AAPRPT+ showed better joint mobilization compared to PRPT+ at D6 $(p=0.03)$, D18 $(p=0.04)$ and D25 $(p=0.02)$. Similar results were found regarding US and histology, with smaller collagen fiber diameters (D6, p $\leq 0.017$, D25, $p \leq 0.015)$, less disorganization and fewer neovessels (D25, $p=0.004)$ in AAPRPT+ compared to PRPT+. No AA+PRP local toxicity was discovered in histology assessment.

Conclusion: our study suggests that combined injection of AA and PRP in tendinosis accelerates and improves tendon's healing compared PRP used alone, with no local toxicity.

KEY WORDS: anti-angiogenic, PRP, rat, tendon, tendinosis, US.

\section{Introduction}

Tendinosis $(\mathrm{T}+)$ is a very common and disabling condition, resulting in impairment of quality of life. Indeed, $T+$ of the rotator cuff affects $3-20 \%$ of the general population, mainly women between 40 and 65 year old whereas Achille's T+ affects $5-6 \%$ of the general population, especially young men. In most cases, this condition progresses to a disabling pain or tendon rupture, which can impact personal and professional activities ${ }^{1}$.

The healthy tendon is principally composed of type 1 collagen and a few elastic fibers, within a ground substance containing cells (tenocytes and tenoblasts) and water. In case of $\mathrm{T}+$, histology shows thinned and disorganized collagen fibers, mucoid and/or lipoïd degeneration and increased interfibrillar glycosaminoglycans deposition ${ }^{2}$. In addition to these lesions, neo-angiogenesis and nerve fiber development have been reported at the beginning of $T+$ and throughout tendon healing ${ }^{3}$. Inflammatory lesions are rare, but may be associated with tendon rupture ${ }^{1}$. Early treatment of $\mathrm{T}+$ should therefore be recommended to avoid complications. 
Several lines of research have been explored for the curative treatment of $\mathrm{T}+$ and tendon rupture, including Ultrasound (US)-guided fenestration or tenotomy 4,5 , and intratendinous injections of hyperosmolar solutions $^{5}$, bone morphogenic protein ${ }^{6}$, sclerosis product $^{7}$, anti-angiogenic therapies $(A A)^{8}$ or platelet-rich plasma (PRP) with varying efficiencies ${ }^{9,10}$.

PRP is defined as serum with a platelet concentration from 3 to 8 times higher than in blood, which permits the availability of more important concentrations of active growth factors (PDGF, TGF- $\beta$, VEGF...) stimulating healing. PRP promotes stem cell recruitment and directly stimulate collagen production by the tendon tenoblasts ${ }^{9}$. PRP can be directly injected into tendons to enhance local platelet concentration ${ }^{10}$. Numerous in vitro ${ }^{11,12}$ and animal studies using this technique have been performed in animal models with results demonstrating improvement of clinical and histological repair ${ }^{13,14}$.

Anti-angiogenic therapies (AA) can also provide a therapeutic effect by simultaneously inducing neovessel definitive vasoconstriction and inhibiting the development of neovessels in the tendon ${ }^{8}$.

To our knowledge, no studies have been performed to evaluate combined injection of AA and PRP by inhibiting neovessels for $A A$ and stimulating collagen production for PRP, in case of $T+$. The aim of our study was therefore to assess the potential of combined intratendinous injection of $A A$ and PRP to treat $\mathrm{T}+$ in a rat model of patellar and Achilles $\mathrm{T}+$, and to evaluate its local toxicity.

\section{Materials and methods}

The procedures and the animal care complied with the "Principles of animal care" formulated by the European Union (Animal Facility Agreement 75-18-03, 2005), and animal experimentation was performed under the authorization 75-101 of the French Ministry of Agriculture.

Twenty immunocompetent healthy male Sprague Dawley rats (providing 40 patellar and 40 Achilles tendons, age $=10$ weeks old, mean weight $=252 \mathrm{~g} \pm$ 32, CERJ, Le Genest, France) were used. The rats were sedated before and during each manipulation with Isoflurane ${ }^{\circledR}$ ( $5 \%$ for induction and $2.5 \%$ for maintenance) ${ }^{15}$.

During follow-up, rats were housed in groups of 4 in stalling cages at the INSERM U698 conventional animal housing facility. Animals were submitted to a standard laboratory rat pellets with water at libitum, with a $12 \mathrm{~h}$ light/dark cycle (12h light $12 \mathrm{~h}$ dark) and a $20 \pm 2^{\circ} \mathrm{C}$ temperature.

At D0, we (LL) induced chemical $\mathrm{T}+$ on all 80 patellar and Achilles normal and asymptomatic tendons under Ultrasonography (US) guidance after initial clinical and US evaluation, by a single intra-tendinous injection of Type 1 Collagenase Gibco $^{\mathrm{TM}}$ (250 U ie $30 \mathrm{mi}-$ crol, dissolved in 0,09\% saline solution PROAM®, using a $29 \mathrm{G}$ needle). This model of $\mathrm{T}+$ has been described in previous publications and provides an ani- mal model of $T+$ as early as 3 days after collagenase injection and thereafter, up to 12 weeks $^{16,17}$. At D3, after clinical assessment, we initiated $T+$ treatment. Treatment consisted of an intratendinous injection under US guidance (targeting the thickened segment of the tendon) using a 29G needle of either $0,05 \mathrm{ml}(1,25$ $\mathrm{mg}$ ) of AA followed by $0,05 \mathrm{ml}$ of PRP (AAPRP+) or a single injection of $0,1 \mathrm{ml}$ of PRP (PRPT+). All left paw were treated with $A A$ and $P R P$, all right paw were treated with PRP only. We chose to inject a maximum of $0.1 \mathrm{ml}$ in each tendon, as a higher dose would have damaged these small size tendons.

Technically, the needle was inserted in the tendon with an angulation of 45 degrees, and the injection of either collagenase, AA + PRP or PRP was performed once the needle reached the middle of the tendon. No specific regimen or restricted activity followed the intratendinous injection. Similar to previous work on corneal neovascularization, we chose high dose of bevacizumab (Avastin $\AA$ ) to obtain antiangiogenic effect $^{8}$. Regarding PRP preparation, we performed the following steps: with drawal of the rat's peripheral jugular blood $(3 \mathrm{ml})$ and a single 8 minutes spin centrifugation (1800 tour/min) with no activator to obtain a final volume of PRP (visible as a yellow layer) of 1 $\mathrm{ml}$. This PRP obtained had a platelet concentration equal to 3 times (mean $=1.500 .000 \pm 42.000$ ) the concentration measured in the blood as verified using a conventional cytometry method by Scil vet abc Plus ${ }^{\circledR}$. We chose this concentration, as it is the lowest accepted platelet concentration for PRP in definition. Our PRP preparation was also poor in leukocytes which were also counted by the same cytometry method (mean $=1300 \pm 1.00$ ) to avoid any acute inflammatory response with catabolic effects ${ }^{10,18,19}$.

Among these 80 patellar and Achilles tendons, efficacy of AA and PRP was studied by comparing AAPRPT+ and PRPT+ at D6, D18 and D25. At D6, clinical and US examination were performed on 40 AAPRPT + and 40 PRPT+ (20 patellar and 20 Achilles tendons). At D18, clinical and US examination were performed on the 36 remaining PRPT+ (one rat died at D8) and 40 AAPRPT+. At D25, clinical and US examinations were performed with additional histology on the 36 remaining PRPT+ and 40 AAPRPT+.

Clinical evaluation (BD, OZ) of each tendon was based on a semi-quantitative clinical score (Score 0 : normal mobility, Score 1: moderate mobility of the paw, Score 2: no mobility of the paw). It was performed via observation of the animal within their cage by two independent operators, blindly to treatment knowledge and recorded after consensus. US examination (BD, OZ) was performed using a high frequency ultrasound (US) VEVO 2100 (VisualSonic $\left.{ }^{\circledR}\right)$ apparatus dedicated to small animals, with a $50 \mathrm{MHz}$ transducer (spatial resolution: 30 micron). We measured tendon antero-posterior diameters (on longitudinal cut) on straight tendons (obtained by dynamic joint flexion), similar to validated human and animal model US tendon measurement methods ${ }^{16,20}$. The transducer was positioned in the visually thickened segment of the tendon. All measurements were also 
performed by two independent operators blindly to treatment knowledge and recorded after consensus. This US measurement method was previously validated in our institution on a rat model before we initiated our study (unpublished data) with intra-reader variability of $0.05 \mathrm{~mm}$ on patellar and Achilles tendon. Fixating tissue samples with formalin for 48 hours, embedding in paraffin, and staining $5 \mu \mathrm{m}$ sections with Hematoxylin and Eosin (HE) performed histology (LD). The actually Bonar score in the $\mathrm{T}+$ targeting area was used to assess changes in tendon histology, blindly to treatment knowledge by the anatomopathologist (LD). Using four grades (0 to 3), it evaluated tenocytes (nucleus form and cytoplasm quantity), ground substance (quantity and aspect), collagen fibers organization and vascularity quantity between bundles ${ }^{21}$.

Regarding the toxicity of combined injection of AA and PRP, we searched for abnormal cellular infiltrates or necrosis on histology.

\section{Statistical analysis}

Statistical analysis was performed using SAS $®$ software 9.2. To study the efficacy of combined injection of AA and PRP, we compared clinical scores and US evaluation (anteroposterior diameters of patellar and Achilles PRPT+ and AAPRPT+ in longitudinal cuts) at day 6 , day 18 and day 25 , using a Wilcoxon rank-sum test, for non-normal distributions. The two groups were considered as independent.

Then, the same test was used to compare histological scores in the different treatment groups at D25. We considered $p<0.05$ as significant.

\section{Results}

After intratendinous collagenase injection, during clinical follow up, all animals showed a persistent deterioration of their ability to mobilize $T+$ at day 3 (mean: 2, SD: 1). For efficacy of combined injection of AA and PRP to treat T+, during follow up, at D6, D18 (for Achilles tendon only), and D25, AAPRPT+ had a better clinical score than PRPT+ $(\mathrm{p}<0.05)$.

At D6, US showed smaller anteroposterior diameters in the patellar in the AAPRPT+ group compared to the PRPT+ group $(p=0,017)$ with similar results in Achilles tendons $(p<0,001)$ suggesting synergy between $A A$ and PRP when injected successively (Fig. 1, Tab. 1).

At D18, US showed no difference between anteroposterior diameters in the patella in the AAPRPT+ group compared to the PRPT+ group $(p=0,225)$ with however efficient results in Achilles tendons $(p<0,001)$ (Tab. 1).

At D25, US showed smaller anteroposterior diameters in the patella and Achilles tendons in the AAPRPT+ group compared to the PRPT+ group $(p=0,015$ and $p<0,001$ respectively) suggesting effectiveness of combined injection of AA and PRP (Tab. 1).

Histological examination performed on 40 AAPRPT+ and 36 PRPT+ at D25 showed less fibrillar disorganization and neovascularisation in AAPRPT+ group (mean Bonar score: 3, SD 0) versus the PRPT+ group (mean Bonar score: 5 , SD 1) $(p=0,004)$. Histology showed absence of macrophages in the 2 groups (Fig. 2).

About the toxicity of combined injection of $A A$ and PRP, we found no abnormal cellular infiltrates or necrosis in HE histology.

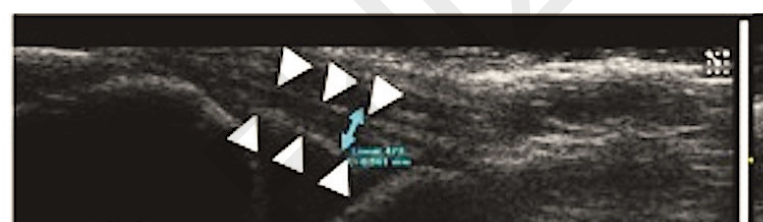

tibia

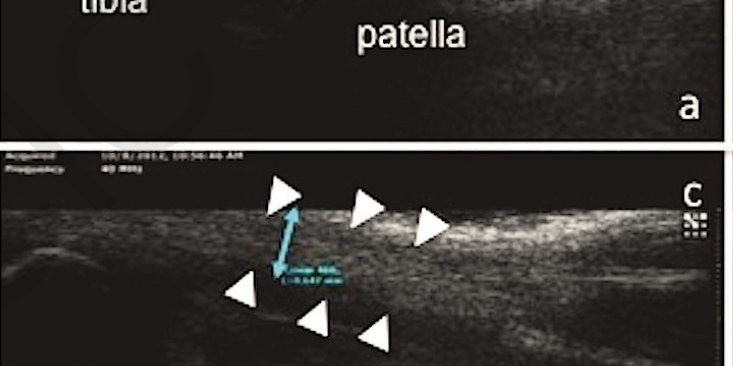

calcaneus
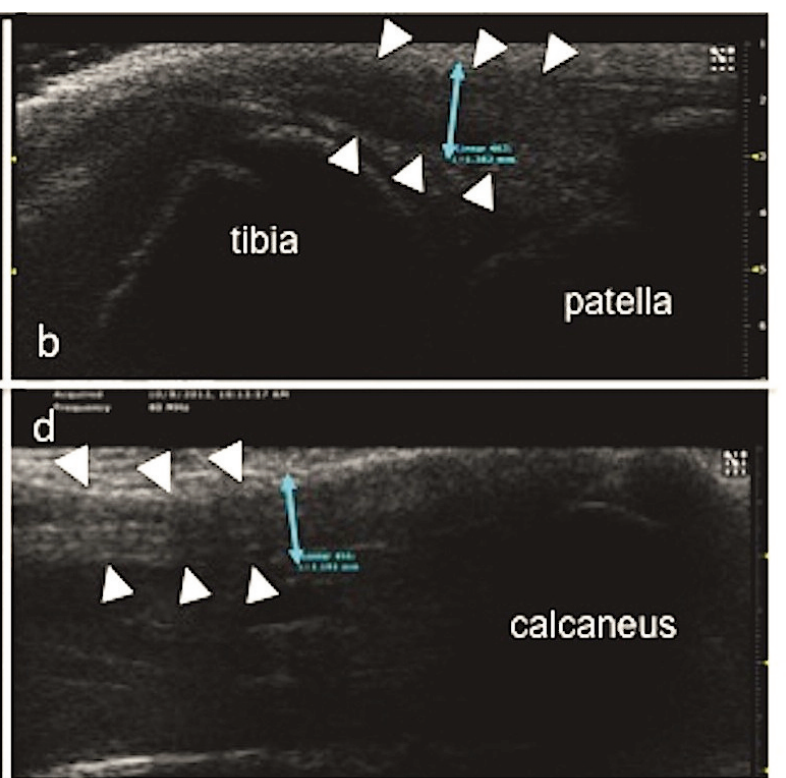

Figure 1. US sagittal slice of normal (D0) and tendinosis (D6) patellar tendon (a, b) and Achilles tendon (c, d) (arrowheads). 
Table 1. Mean and SD (millimeters) values of US lesions in each anatomic compartment in patellar and Achilles tendon at Day 6 (D6), Day 18 (D18) and Day 25 (Day 25). $\left(\left\langle=p=0,017 ;^{\circ}=p<0,01 ;{ }^{*}=p=0,225 ;^{* *}=p=0,015\right)\right.$.

\begin{tabular}{|c|c|c|c|c|c|c|}
\cline { 2 - 7 } \multicolumn{1}{c|}{} & \multicolumn{2}{c|}{ D6 } & \multicolumn{2}{c|}{ D18 } & \multicolumn{2}{c|}{ D25 } \\
\cline { 2 - 7 } & PRP & PRP+AA & PRP & PRP+AA & PRP & PRP+AA \\
\hline $\begin{array}{c}\text { Patellar tendon } \\
(\mathrm{mm})\end{array}$ & $1,45+/-0,01$ & $1,1+/-0,03 \cdot$ & $1,1+/-0,01$ & $1,0+/-0,04^{*}$ & $1,0+/-0,01$ & $0,9+/-0,01^{* *}$ \\
\hline $\begin{array}{c}\text { Achille tendon } \\
(\mathrm{mm})\end{array}$ & $1,24+/-0,01$ & $0,8+/-0,01^{\circ}$ & $1,1+/-0,01$ & $0,7+/-0,01^{\circ}$ & $0,95+/-0,01$ & $0,75+/-0,01^{\circ}$ \\
\hline
\end{tabular}

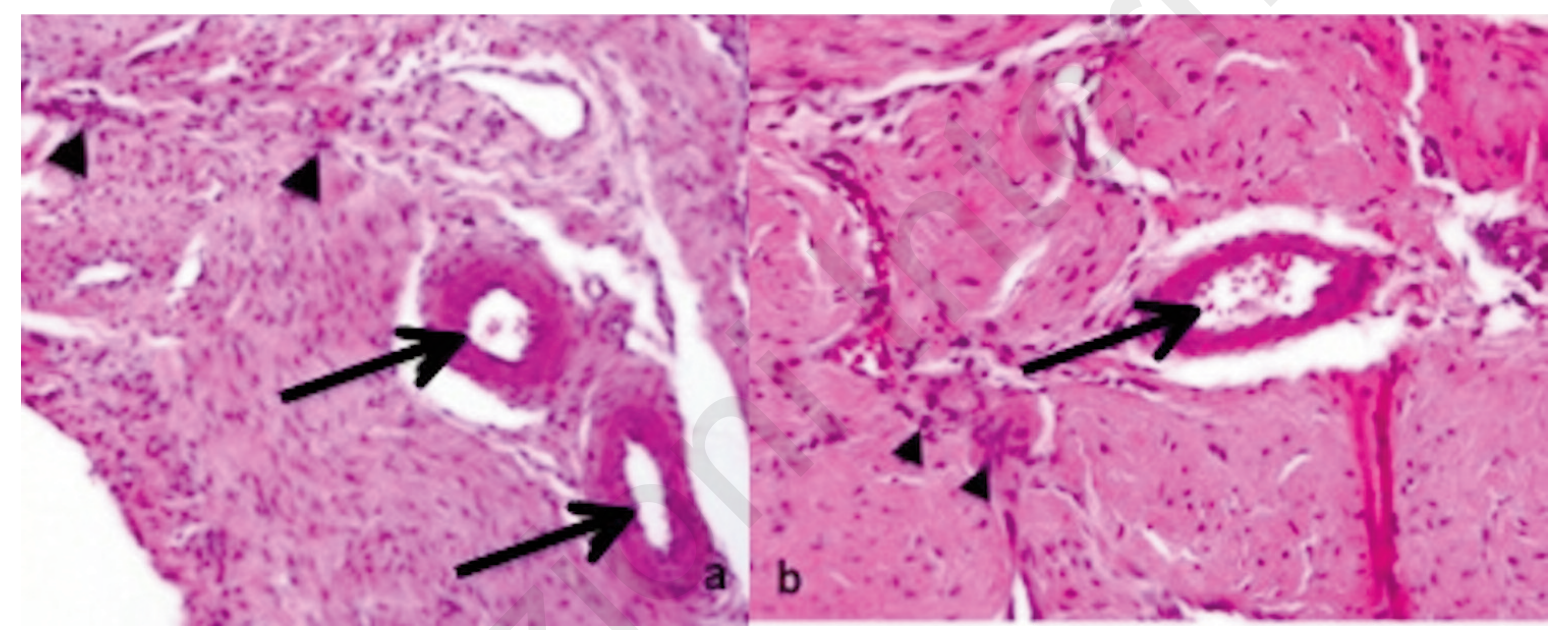

Figure 2. PRPT+ (a: magnification: $x$ 200) with Bonar score 5 and AAPRPT+ (b: magnification: $x$ 200) with Bonar score 3 Achilles enthesis showing collagen dizorganisation (arrowheads) with neovascularisation (arrows) at D25.

\section{Discussion}

Our study strongly suggests that a single intratendinous US-guided injection of AA and PRP in Achilles and patellar $\mathrm{T}+$ accelerates and improves the quality of $\mathrm{T}+$ healing compared to a control PRPT+ group, with no local toxicity. These results are potentially important as, to our knowledge, there has been no study demonstrating the curative effect of combined injection of $A A$ and $P R P$, early in the evolution of tendinosis, before tendon rupture and before the onset of chronic pain. Physiologically, a tendon is formed by collagen fibers. In $T+$ rats, histology showed early anarchic misalignment of collagen fibers, resulting in important fibrillar disorganization after induction of $T+{ }^{1}$. Importantly, reports show no sign of inflammation in $\mathrm{T}+$. Our histology and immunohistochemical study confirmed these results with the presence of fiber disorganization, intratendinous neoangiogenesis and absence of inflammatory cells. Regarding neoangiogenesis, it has been reported that neovessels, early after the beginning of $\mathrm{T}+$, carries proteolytic enzymes, nitric oxide and deleterious prostaglandins that may be responsible for tendon degeneration ${ }^{22}$. Conversely, later in the healing process, these neovessels provide active growth factors that stimulate healing and leukocyte recruitment. These growth factors promote stem cells and directly stimulate fibroblast-mediated collagen production ${ }^{23}$. Therefore, AA injected early after $\mathrm{T}+$ induction might act positively by preventing deleterious proteolytic enzymes and prostaglandins, but might preclude local increase of active growth factors therefore limiting scarring ${ }^{8}$.

Intratendinous PRP injection might compensate for neo-vessel destruction by locally providing important concentration of active growth factors (PDGF, TGF- $\beta$, VEGF...), therefore promoting stem cell recruitment and fibroblast collagen production which stimulate tendon healing 24 . 
Recently, we performed two pre-clinical studies to assess the efficacy of intra-tendinous injection of PRP used alone and AA used alone to treat tendinosis comparing to control group represented by intra tendinous injection of physiological serum (PS) alone $^{8}$. In these studies, we used the same setup, animal model, and controlled PRP and AA preparation protocol as described in this study. Our results demonstrated that both PRP and AA could shorten $T+$ healing process and that PRP could also improve the quality of $T+$ healing. Based on these results and the potential complementarity between AA and PRP to avoid catabolic effects and stimulate thrombus in tendon as described above, our next step was therefore to assess a potential synergetic effect of AA and PRP to shorten and improve the quality of $T+$ healing. By assessing combined injection of AA and PRP in a rat model, with a systematic clinical and US follow-up and histological examination, our study provides strong evidence that AA+PRP might be a useful strategy to treat $T+$ as it accelerates and improves the quality of tendon healing.

Higher doses of $\mathrm{PRP}^{25}$ or $\mathrm{AA}$, injection with tenotomy, could equally have been chosen in this study. We chose a fixed and low platelet concentration in PRP (x3) and no adjuvant, to avoid adding to the variety of PRP preparations reported in the literature 26,27 , with PRP platelet concentrations varying between $\times 3$ and $x 8$ compared to the concentration in blood. Regarding the method for PRP and AA injection, we chose to perform intratendinous injection without tenotomy under US guidance as described by Peerbooms et al. or Foster et al. ${ }^{28,29}$, a method which has been shown to induce a significant decrease in tendinopathy in humans. Our study suggests that combined injection of PRP and AA might be useful to reduce recovery time and improve the quality of tendon scarring. This might be particularly useful in top athletes.

Regarding AA, we chose Bevacizumab as it is widely available and as we used it in our pre-clinical study to assess the efficacy of AA used alone to treat tendinosis. However, as gastrointestinal perforation, vascular thrombosis or haemorrhages have been described with high doses in cancer therapies with AA and as $A A$ is not allowed in adolescent and children, additional studies are mandatory before intratendinous injection can be applied to humans. In addition, although we searched for local signs of toxicity after AA intra-tendinous injection in our rat model, we did not search for general signs of toxicity, which would have been beyond the scope of this article. Therefore, until the absence of toxicity for AA can be demonstrated in humans, use of other strategies to destroy neovessels early in the healing process might be useful. The effect of Polidocanol ${ }^{\circledR}$, which is a sclerotic agent, has already been assessed in human patellar tendinopathy, with results demonstrating reduced pain and moderate improvement in knee function? ${ }^{7}$ Combining Polidocanol with PRP might therefore be of interest in the future.

One limitation of our study might be the use of our semi quantitative clinical score to assess the pathological status of tendons, as this score has not validated in the literature. It should be noted however that our results regarding improvement of this score using $A A+P R P$ compared to PRP used alone is coherent with the improvement of tendon thickness measured by US and scarring on histology. Second limitation might be the use of US (not MRI) to assess the pathological status of tendons. However, $50 \mathrm{MHz}$ US transducers provide excellent spatial resolution and allow precise measurement of stretched tendons as well as needle guidance. We also voluntarily chose US rather than small animal MRI as it is faster and equally efficient for thickness measurements ${ }^{1}$ especially considering the small intrareader variability $(0.05 \mathrm{~mm}$, non-published data). Finally, compared to histology, US allowed follow up without systematic animal sacrifice. Third, use of a collagenase-based rat model might be a concern as human T+ develops after repeated micro-traumatism. However, collagenase rapidly induces $\mathrm{T}+$, with reproducible lesions and no inflammatory cell infiltration as demonstrated here, which explains its widespread use as a model for $\mathrm{T}+$. Our protocol was based on the literature ${ }^{11}$, which reports tendinosis 3 days after collagenase injection despite effectiveness of tendinopathy induction after injection of collagenase, no US or histology weren't performed at D3 before treatment AA and PRP induction. Fourth, no control group was used in this study because we have already previously proved PRP and AA efficacy in healing tendon process, comparing to control group (PS). Fifth, mechanical properties of the tendons were not performed. Testing the strength of the tendons could be very interesting to correlate with histology. Last, regarding toxicity, it has been suggested that PRP (with low leukocytes concentration) and AA used alone have no local toxicity 8,10 . Similarly, we found no tendon necrosis, rupture or structural modifications on histology (HE) after $A A+P R P$ injection, although no dedicated study on normal tendons nor immunohistology were performed. Additional studies are therefore necessary before the protocol can be applied to humans.

\section{Conclusion}

Our study suggests that a combined injection of $A A$ and PRP in tendinosis using a controlled concentration of platelets and leukocytes accelerates and improves tendon healing in rats, according to clinical, US and histological criteria, with no local toxicity. Additional pre-clinical studies optimizing the injection protocol (time of injection and dose) and comparing PRP+AA to currently used methods might be of high interest to confirm the potential of combined intra tendinous injection of $A A$ and PRP, before it can be applied to human.

\section{References}

1. Paavola M, Kannus $P$, Jarvinen TA, Khan $K$, Jozsa L, Jarvinen M. Achilles tendinopathy. J Bone Joint Surg Am. 2002;84A:2062-2076. 
2. Khan KM, Maffulli N. Tendinopathy: an Achilles' heel for athletes and clinicians. Clin J Sport Med. 1998;8:151-154.

3. Nelen G, Martens M, Burssens A. Surgical treatment of chronic Achilles tendinitis. Am J Sports Med. 1989;17:754-759.

4. Housner JA, Jacobson JA, Morag Y, Pujalte GG, Northway RM, Boon TA. Should ultrasound-guided needle fenestration be considered as a treatment option for recalcitrant patellar tendinopathy? A retrospective study of 47 cases. Clin J Sport Med. 2010;20:488-490.

5 Rabago D, Best TM, Zgierska AE, Zeisig E, Ryan M, Crane D. A systematic review of four injection therapies for lateral epicondylosis: prolotherapy, polidocanol, whole blood and platelet-rich plasma. Br J Sports Med. 2009;43:471-481.

6. Park EJ, Kim ES, Weber HP, Wright RF, Mooney DJ. Improved bone healing by angiogenic factor-enriched plateletrich plasma and its synergistic enhancement by bone morphogenetic protein-2. Int J Oral Maxillofac Implants. 2008;23:818826.

7. Coombes BK, Bisset L, Vicenzino B. Efficacy and safety of corticosteroid injections and other injections for management of tendinopathy: a systematic review of randomised controlled trials. Lancet. 2010;376:1751-1767.

8. Dallaudiere B, Lempicki M, Pesquer L, et al. Acceleration of tendon's healing using guided intratendinous injection of bevacizumab: first pre clinical study on a murine modele. European journal of radiology. 2013; in press.

9. Finnoff JT, Fowler SP, Lai JK, et al. Treatment of chronic tendinopathy with ultrasound-guided needle tenotomy and platelet-rich plasma injection. PM R. 2011; 3:900-911.

10. Dallaudiere B, Lempicki M, Pesquer L, et al. Efficacy of intratendinous injection of platelet-rich plasma in treating tendinosis: comprehensive assessment of a rat model. Eur Radiol. 2013; in press.

11. Carofino B, Chowaniec DM, McCarthy MB, et al. Corticosteroids and local anesthetics decrease positive effects of platelet-rich plasma: an in vitro study on human tendon cells. Arthroscopy. 2012;28:711-719.

12. GeburekF, Stadler P. Regenerative therapy for tendon and ligament disorders in horses. Terminology, production, biologic potential and in vitro effects. Tierarztl Prax Ausg G Grosstiere Nutztiere. 2011;39:373-383.

13. Kaux JF, Drion PV, Colige A, et al. Effects of platelet-rich plasma (PRP) on the healing of Achilles tendons of rats. Wound Repair Regen. 2012;20:748-756.

14. Kaux JF, Drion P, Libertiaux V, et al. Eccentric training improves tendon biomechanical properties: a rat model. J Orthop Res. 2013;31:119-124.

15. Padulo J, Oliva F, Frizziero A, Maffulli N. Muscles, Ligaments and Tendons Journal. Basic principles and recommenda- tions in clinical and field science research. MLTJ. 2013;4: 250-252.

16. Lui PP, Fu SC, Chan LS, Hung LK, Chan KM. Chondrocyte phenotype and ectopic ossification in collagenase-induced tendon degeneration. J Histochem Cytochem. 2009;57:91-100.

17. Warden SJ. Animal models for the study of tendinopathy. Br J Sports Med. 2007;41:232-240.

18. Dragoo JL, Braun HJ, Durham JL, et al. Comparison of the acute inflammatory response of two commercial platelet-rich plasma systems in healthy rabbit tendons. Am J Sports Med. 2012;40:1274-1281.

19. McCarrel TM, Minas T, Fortier LA. Optimization of leukocyte concentration in platelet-rich plasma for the treatment of tendinopathy. J Bone Joint Surg Am. 2012;94:e143(141-148).

20. Scott A, Sampaio A, Abraham T, Duronio C, Underhill TM Scleraxis expression is coordinately regulated in a murine model of patellar tendon injury. J Orthop Res. 2011;29:289296.

21. Maffulli N, Longo UG, Franceschi F, Rabitti C, Denaro V. Movin and Bonar scores assess the same characteristics of tendon histology. Clin Orthop Relat Res. 2008;466:16051611.

22. Alfredson $\mathrm{H}$, Lorentzon $\mathrm{M}$, Backman $\mathrm{S}$, Backman $\mathrm{A}$, Lerner UH. cDNA-arrays and real-time quantitative PCR techniques in the investigation of chronic Achilles tendinosis. J Orthop Res. 2003;21:970-975.

23. Vu TH, Werb Z. Matrix metalloproteinases: effectors of development and normal physiology. Genes Dev. 2000;14:21232133.

24. Sharma $P$, Maffulli N. Tendon injury and tendinopathy: healing and repair. J Bone Joint Surg Am. 2005;87:187-202.

25. Kampa RJ, Connell DA. Treatment of tendinopathy: is there a role for autologous whole blood and platelet rich plasma injection? Int J Clin Pract. 2010;64:1813-1823.

26. de Vos RJ, van Veldhoven PL, Moen MH, Weir A, Tol JL, Maffulli N. Autologous growth factor injections in chronic tendinopathy: a systematic review. Br Med Bull. 2010;95:6377 .

27. Murawski CD, Kennedy JG. Platelet-rich plasma releasate promotes differentiation of tendon stem cells into active tenocytes. Letter to the editor. Am J Sports Med. 2010;38:NP3.

28. Peerbooms JC, Sluimer J, Bruijn DJ, Gosens T. Positive effect of an autologous platelet concentrate in lateral epicondylitis in a double-blind randomized controlled trial: platelet-rich plasma versus corticosteroid injection with a 1-year follow-up. Am J Sports Med. 2010;38:255-262.

29. Foster TE, Puskas BL, Mandelbaum BR, Gerhardt MB, Rodeo SA. Platelet-rich plasma: from basic science to clinical applications. Am J Sports Med. 2009;37:2259-2272. 